\title{
AVALIAÇÃo DE TÉCNICAS DE SEGMENTAÇÃO E CLASSIFICAÇÃO AUTOMÁTICA DE IMAGENS LANDSAT-TM NO MAPEAMENTO DO USO DO SOLO NA AMAZÔNIA'
}

\author{
Raimundo ALMEIDA FILHO ${ }^{2}$, Paulo Sérgio Rezende NASCIMENTO², Getulio \\ Teixeira BATISTA ${ }^{2}$
}

RESUMO - O mapeamento do uso da terra é fundamental para o entendimento dos processos de mudanças globais, especialmente en regiões como a Amazônia que estão sofrendo grande pressão de desenvolvimento. Tradicionalmente estes mapeamentos têm sido feitos utilizando técnicas de interpretação visual de imagens de satélites, que, embora de resultados satisfatórios, demandam muito tempo e alto custo. Neste trabalho é proposta uma técnica de segmentação da imagens com base em um algoritmo decrescimento de regiões, seguida de uma classificação não-supervisionada por regiôes. Desta forma, a classificação temática se refere a um conjunto de elementos (pixels da imagem), beneficiando-se portanto da informação contextual e minimizando as limitações das técnicas de processamento digital baseadas em análise pontual (pixela-pixel). Esta técnica foi avaliada numa área típica da Amazônia, situada ao norte de Manaus, AM, utilizando imagens do sensor "Thematic Mapper" - TM do satẻlite Landsat, tanto na sua forma original quanto decomposta em elementos puros como vegetação verde, vegetação seca (madeira), sombra e solo, aqui denominada imagem misturas. Os resultados foram validados por um mapa de referência gerado a partir de técnicas consagradas de interpretação visual, com verificação de campo, e indicaram que a classificação automática é viável para o mapeamento de uso da terra na Amazônia. Testes estatísticos indicaram que houve concordância significativa entre as classificações automáticas digitais e o mapa de referência (em torno de $95 \%$ de confiança).

Palavras-chave: Mapeamento temático automatizado, segmentação de imagens, classificação nãosupervisionada por regiões, mudanças no uso da terra, sensoriamento remoto.

\section{Evaluation of Segmentation and Automatic Classification Techniques of Landsat - TM Imagery for Land Use Mapping in Amazonia.}

ABSTRACT - Land use mapping is essential for the understanding of global change processes, especially in regions which are experiencing great pressure for development such as the Amazon. Traditionally, these mappings have been done using visual interpretation techniques of satellite imagery, that provide satisfactory results but are time-consuming and highly cost. In this paper, a technique of image segmentation based on region growing algorithm, followed by a per-field non-supervised classification, is proposed. Thus, the thematic classification is based on a set of image elements (pixels), benefiting from contextinformation, therefore minimizing the limitations of the digital processing techniques based on single pixels (per-pixel classification). This approach was evaluated in a typical test site of the Amazon region located to the north of Manaus, AM, using both original Landsat Thematic Mapper images and their decomposition into endmembers such as green vegetation, wood material, shade and soil, named mixture image in this paper. The results were validated by a reference map obtained from proved visual interpretation techniques of satellite imagery and by field check and indicated that automatic classification is feasible to map land use in Amazônia. Statistics tests indicated that there was significant agreement between the automated digital classifications and the reference map (at 95\% confidence level).

Key-words: Automated thematic mapping, image segmentation, per-field non-supervised classification, land use change, remote sensing.

Parte da Dissertação de Mestrado de Paulo Sérgio de R. Nascimento, desenvolvida no Curso de Pós-graduação em Sensoriamento Remoto do Instituto Nacional de Pesquisas Espaciais (INPE), com apoio da Coordenação de Aperfeiçoamento de Pessoal de Nivel Superior (CAPES), sob a orientação dos demais autores.

2 DSR/INPE, Av. dos Astronautas, 1758, 12.227-010 - São José dos Campos, SP. 


\section{INTRODUÇÃO}

O emprego de imagens de sensoriamento remoto orbital no mapeamento e monitoramento do uso da terra, especialmente quando envolvendo grandes áreas, tem sido baseado no uso de técnicas de interpretação visual. Em tais casos esse procedimento envolve grande consumo de tempo e requer a participação de vários especialistas nas suas diferentes etapas. Um exemplo são os levantamentos conduzidos pelo Instituto Nacional de Pesquisas Espaciais-INPE (Tardin \& Cunha, 1989; INPE, 1996) sobre desflorestamento na Amazônia. Para cada levantamento cobrindo a região Amazônica são interpretadas cerca de 230 composiçóes coloridas do satélite Landsat na escala de 1:250.000, com a conseqüente geração de igual número de overlays. Todo o procedimento envolve várias etapas até a geração dos mapas finais: interpretação das imagens; ajustes entre overlays de diferentes intérpretes; digitalização de mapas, correção e ajuste dos dados, etc. A automatização ou semi-automatização de levantamentos como esse implicaria em ganhos em termos de precisão, redução de tempo e custos. Entretanto, a opção pela interpretação visual decorre do baixo desempenho das técnicas de classificação automática convencionais (pixel a pixel) ainda em uso.

De modo a contornar essas limitações, grande esforço tem sido colocado no desenvolvimento e aplicação de novos métodos de classificação automática, os quais, à semelhança de um fotointérprete, possam incorporar informações espaciais da cena e não considerar isoladamente as informações pontuais contidas nas imagens de sensoriamento remoto. A aplicação de tais técnicas no mapeamento de áreas desflorestadas na Amazônia tem mostrado resultados animadores que permitem antever o uso generalizado de tais abordagens em levantamentos regionais, como atestam os trabalhos pioneiros de Batista et al. (1994), Santos et al. (1995), Machado et al. (1994) e Alves et al. (1996).

Dentro desse contexto, o presente trabalho tem como objetivo avaliar e quantificar o desempenho de técnicas de segmentação e classificação automática nãosupervisionada por regiões, no mapeamento do uso da terra em uma área teste na Amazônia, utilizando diferentes produtos gerados a partir de imagens do Landsat Thematic Mapper (TM), comparando-se os resultados com um mapa obtido por fotointerpretação convencional.

\section{CARACTERÍSTICAS GERAIS DA ÁREA DE ESTUDO}

A área de estudo localiza-se a cerca de $70 \mathrm{~km}$ ao norte de Manaus (AM), cobrindo uma superfície de aproximadamente $190 \mathrm{~km}^{2}$, delimitada pelas coordenadas de $2^{\circ} 19^{\prime} 13^{\prime \prime}$ e $2^{\circ}$ 25 ' 09" de latitude Sul e 60 $06^{\prime}$ '03" e 59 $58^{\prime} 24^{\prime \prime}$ de longitude Oeste (Fig. 1). $\mathrm{O}$ acesso é feito pela rodovia BR174, que liga Manaus a Boa Vista, em Roraima. $\mathrm{O}$ clima na região é quente e úmido, com temperaturas médias 
anuais acima de $25^{\circ} \mathrm{C}$, e precipitações pluviométricas anuais superiores a $2.000 \mathrm{~mm}$, com maior concentração entre outubro e maio (Projeto RADAMBRASIL, 1978). O relevo na área de estudo é plano a levemente ondulado, ocorrendo predominâncias de latossolos amarelos de baixa fertilidade. A cobertura vegetal na região é a Floresta Tropical Densa, com grande diversificação e densidade de espécimes por unidade de área. Nas áreas desmatadas ocorrem manchas de Formações Pioneiras, em fase de regeneração.

Nos anos 70 a região foi palco de intenso processo de ocupação do solo, com desmatamentos para implantação de pastagens. Entretanto, já no final dos anos 80 , as áreas ocupadas encontravam-se praticamente abandonadas e tomadas por capoeiras. Sob esse aspecto, a área de estudo é bastante representativa do que tem ocorrido em outras áreas da Amazônia. Essas características, aliadas ao fácil acesso que permite verificações de campo, foram fatores que justificaram a escolha da área para estudo.

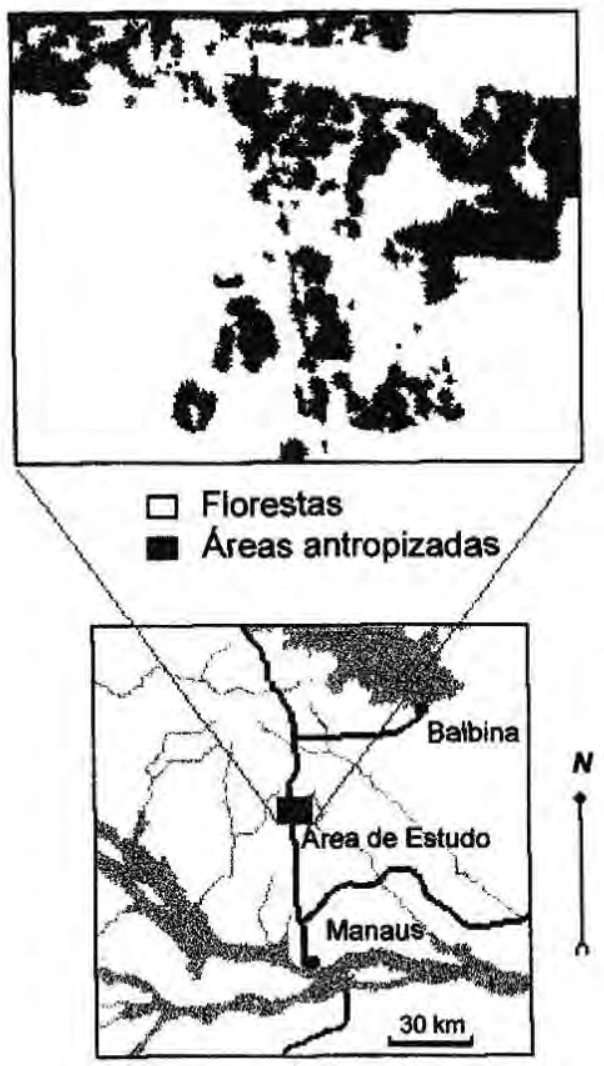

Figura 1. Localização da área de estudo. 


\section{ABORDAGEM METODOLÓGICA}

No estudo foram utilizadas imagens Landsat-TM correspondentes à órbita 231 , ponto 63 , obtidas em 02 de agosto de 1989. Antes dos processamentos digitais as imagens foram corrigidas geometricamente e transformadas para a projeção Universal Transversa de Mercator, usando-se como referência cartas plano-altimétricas na escala de 1:100.000, folhas Fazenda Rio Branco (SA.20-Z-B-III) e Rio Urubu (SA.21-Y-A-I), produzidas pelo Diretoria de Serviços Geográficos do Ministério do Exército. Todos os processamentos digitais foram desenvolvidos no Laboratório de Processamento Digital de Imagens do Instituto de Pesquisas Espaciais-INPE, em São José dos Campos, exceto aqueles relativos à obtenção de imagens misturas, que foram executados no Laboratório de Sensoriamento Remoto do Instituto de Geologia da Universidade de Washington, em Seattle, USA.

Três mapas temáticos mostrando o uso e ocupação do terreno na área de estudo, foram gerados a partir de produtos oriundos de diferentes processamentos digitais das imagens Landsat. $\mathrm{O}$ primeiro mapa temático foi gerado a partir de fotointerpretação, utilizando uma composição colorida Landsat, obtida com as bandas TM3, TM4 e TM5, combinadas com filtros azul, verde e vermelho, respectivamente. Esse tipo de produto tem sido comumente utilizado em estudos conduzidos pelo INPE visando levantamentos de áreas de desflorestamentos na Amazônia (INPE, 1996). A interpretação visual foi realizada analisando-se diferentes elementos nas imagens como cor, forma, tamanho, textura etc., utilizando-se o "cursor" da mesa digitalizadora para traçar as unidades interpretadas. Este produto foi considerado um mapa padrão que serviria como parâmetro de referência para avaliação da precisão e performance dos outros dois mapas temáticos, gerados por técnicas de segmentação e classificação automática não-supervisionada.

Para a obtenção dos mapas temáticos a partir de classificação automática foram utilizados dois tipos de produtos: as mesmas três bandas utilizadas na interpretação visual e imagens misturas, as quais têm se mostrado bastante eficientes para $o$ mapeamento e monitoramento de unidades de paisagem na Amazônia, como mostram os trabalhos de Adams et al. $(1990 ; 1995)$. Neste tipo de produto as imagens originais são decompostas de modo a expressar as frações de alguns constituintes da superfície do terreno, responsáveis pela maioria das informações contidas em cada elemento de resolução da imagem. Como resultado, as variações de tons não mais relacionam-se com as características espectrais dos componentes do terreno, mas determinam a proporção desses componentes dentro de cada pixel. As imagens Landsat foram decompostas em quatro componentes: sombra, vegetação verde, vegetação seca (madeira) e solo.

Definidos os dois conjuntos de produtos Landsat que seriam analisados, 
passou-se à geração das imagens segmentadas. A segmentação é um processo que permite o particionamento de imagens em regiões homogêneas, descritas por caracteristicas como média, variância, área, perímetro etc. Em geral a segmentação pode ser processada com base em duas características dos tons de cinza de uma imagem: descontinuidade e similaridade. $\mathrm{O}$ método baseado em descontinuidade detecta mudanças abruptas dos níveis de cinza de uma cena. O método da similaridade baseia-se no agregamento de pixels que guardam semelhanças com os vizinhos, cuja similaridade é determinada por uma tolerância $\mathrm{T}$, representada pela distância Euclidiana entre os centros dos segmentos, os quais contêm um único rótulo de identificação. O processo inicia-se a partir de um "pixel-semente" que vai agrupando vizinhos que satisfazem ao critério de similaridade. $\mathrm{O}$ crescimento continua até que sejam encontrados pixels que não satisfazem a esse critério. Uma nova região inicia-se, cuja média inicial é o valor do novo "pixel-semente". A definição do limiar de similaridade é uma etapa critica do processo, pois determinará a densidade da segmentação: se o limiar de similaridade for muito baixo, o processo agregará poucos pixels às regiões; por outro lado, se for muito alto, pixels representativos de muitas regiões serâo incorretamente agrupados. Após vários testes, definiuse o valor 12 para o limiar de similaridade, como aquele que gerava segmentos que melhor representavam a verdade terrestre da área de estudo.

Para maior eficiência do desempenho do processo de segmentação é recomendada a aplicação prévia de um filtro passa-baixa para reduzir a variância dos dados (Nagao \& Matsuyama, 1979). Nesse estudo, aplicou-se antes da segmentação um filtro de mediana (máscara de $3 \times 3$ ), que suaviza a textura das imagens.

Uma vez obtidas as imagens segmentadas, passou-se à fase de classificação temática das regiões, com a aplicação de um algoritmo de classificação não-supervisionada por regiões. Este algoritmo baseia-se em técnicas de "clustering" aplicadas sobre um conjunto de regiões, caracterizadas por atributos estatísticos como média e matriz de covariância (Bins et al., 1992). Ele difere do método de classificação nãosupervisionada convencional, no sentido de que é aplicado sobre um conjunto de regiões obtido a partir da imagem segmentada. $\mathrm{O}$ primeiro passo no processo de classificação é a extração dos atributos estatísticos, a partir das imagens utilizadas para a segmentação. $\mathrm{O}$ algoritmo utiliza o vetor média e a matriz de covariância para estimar o centro das classes, iniciando-se o processo de classificação, dividido em duas etapas. Na primeira etapa são definidos os segmentos que possuem a mesma similaridade estatística, através da definição de um limiar de aceitação. À semelhança do limiar de similaridade, a definição do limiar de aceitação é outro ponto crítico do processo, uma vez que à medida que se diminui esse valor, 
aumenta-se o número de classes geradas. Vários limiares de aceitação foram testados, optando-se pelo de $75 \%$, como o que melhor representava as classes temáticas da área. Na segunda fase do processo (reclassificação) o analista rotula as classes temáticas geradas, podendo unir classes, de modo a expressar mais fielmente a realidade da área de estudo. Cabe ressaltar que, embora executadas pelo computador, as técnicas de classificação por regiões não dispensam a participação do especialista. É ele que instrui, acompanha e avalia os resultados, podendo reiniciar o processo sempre que esses não forem satisfatórios, segundo critérios por ele definidos.

Para avaliar a significância entre os resultados das classificações automáticas utilizou-se a estatística não paramétrica denominada kappa, descrita em Rosenfield \& FitzpatrickLins (1986). O índice kappa vem sendo recomendado (Medeiros, 1987; Cong \& Howarth, 1990) como uma medida apropriada da exatidão entre resultados de classificações por representar inteiramente a matriz de erro com um único indice.

\section{RESULTADOS E DISCUSSÕES}

Como já mencionado, foi gerado um mapa fotointerpretado da área de estudo que mostra as principais unidades de paisagem encontradas. Além do mapeamento dessas unidades, este produto servirá como referência para análise comparativa e avaliação da performance com os produtos gerados por classificação automática nâo-supervisionada, quanto à capacidade dos mesmosem identificar essas mesmas unidades. A Figura 2 mostra o mapa fotointerpretado, onde foram reconhecidas quatro classes de ocupação e uso do terra. De acordo com o mapa, cerca de $71 \%$ da área são cobertos por Floresta Densa, $13 \%$ correspondem a floresta secundárias, constituidas por capoeiras com predominância de $\mathrm{Ce}$ cropia $\mathrm{sp}$; $13 \%$ correspondem a áreas de pastagens, constituídas predominantemente por Brachiaria humidicola; e cerca de $3 \%$ são solos nus, os quais ocorrem geralmente ao longo das estradas e sedes de fazendas. As dificuldades encontradas no processo de fotointerpretação das imagens, foram inversamente proporcionais às dimensões das áreas. Assim, a classe Floresta Densa foi a de mais fácil mapeamento, seguida pela classe Florestas Secundárias. As classes Pastagem e Solo Nu foram aquelas em que se encontrou maior dificuldade na interpretação das imagens, por estarem muitas vezes interligadas, formando padrões texturais complexos. No caso da classe Solo $\mathrm{Nu}$, as dificuldades foram ainda maiores, por essas áreas ocorrerem de forma as vezes irregular com dimensões pequenas, sem padrões definidos para que se pudesse estabelecer chaves interpretativas. As Figuras $3 \mathrm{a}$ e $3 \mathrm{~b}$ mostram aspectos dessas quatro unidades de paisagem, observadas a partir de sobrevôo realizado à época da verificação de campo, em julho de 1990.

As Figuras 4 e 5 mostram os 


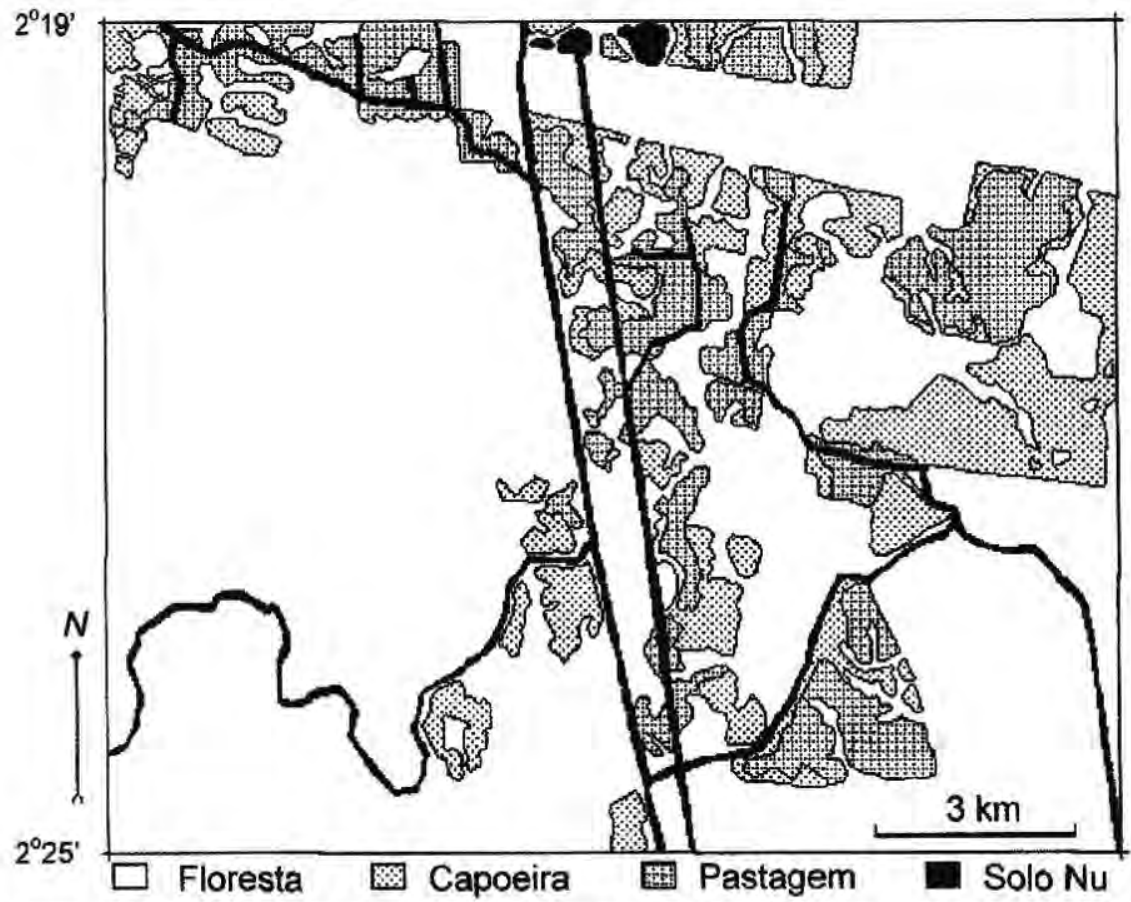

Figura 2. Mapa fotointerpretado a partir de imagens Landsat-TM com as principais unidades de paisagem encontradas na área de estudo.

mapas temáticos obtidos por classificação automática nãosupervisionada, a partir de imagens Landsat originais (bandas TM3, TM4 e TM5) e imagens misturas, respectivamente. Uma simples análise visual comparativa entre os mapas gerados por classificação automática e o mapa fotointerpretado, permite constatar a alta correlação entre esses três produtos, atestando a eficácia das técnicas de segmentação e classificação automática por regiões, como um instrumento de alto potencial como método para o mapeamento semi-automático da ocupação e uso da terra na Amazônia.

De modo a quantificar a análise comparativa entre os três mapas foi realizada uma operação de tabulação cruzada (Star \& Estes, 1990) entre o mapa fotointerpretado e os mapas derivados das classificações automáticas. Este tipo de operação gera uma matriz indicando a correspondencia espacial entre as classes dos mapas considerados.

A Tabela 1 mostra os resultados da análise comparativa entre o mapa fotointerpretado e os mapas temáticos gerados a partir de classificação automática em imagens Landsat normais e imagens misturas. Para a classe Floresta Densa classificada automaticamente, pode-se constatar concordância superior a $94 \%$ com o mapa de referência e apenas cerca de $3 \%$ de confusão entre áreas de florestas primária e secundária 
(a)

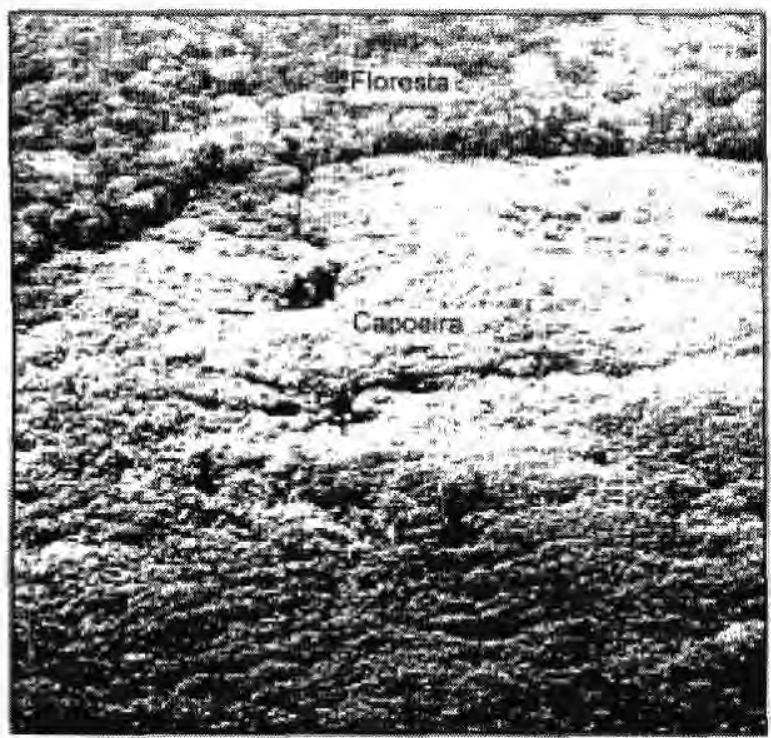

(b)

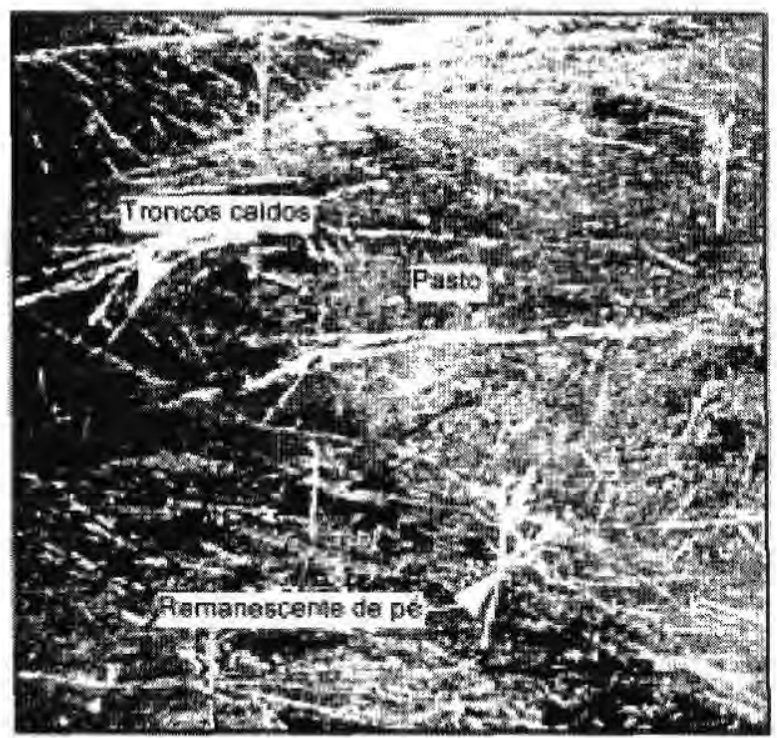

Figura 3. Vistas aéreas da área estudo mostrando áreas de floresta densa e capoeira (a) e área de pastagens (b) (Adaptadas de fotos tomadas por John B. Adams). 


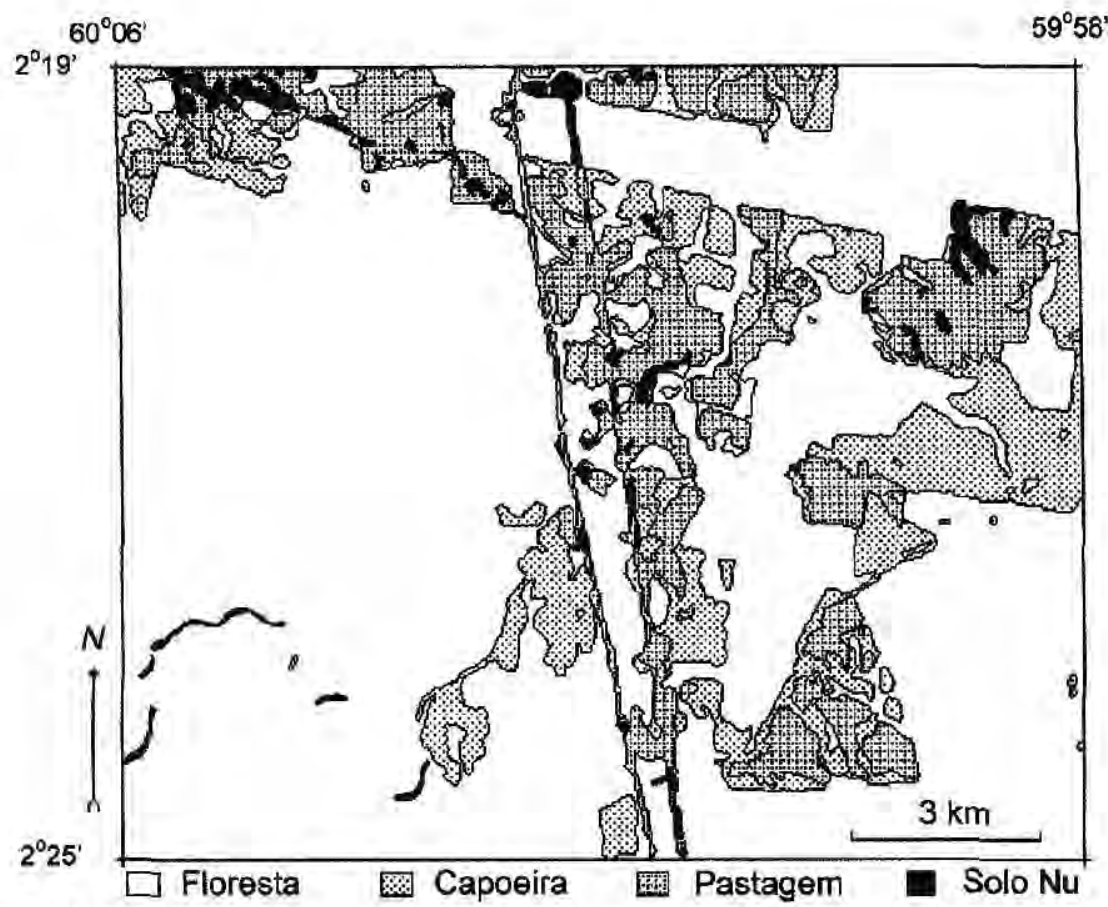

Figura 4. Mapa temático da área de estudo obtido por classificação automática não-supervisionada, à partir de imagens Landsat. bandas TM3, TM4 e TM5.

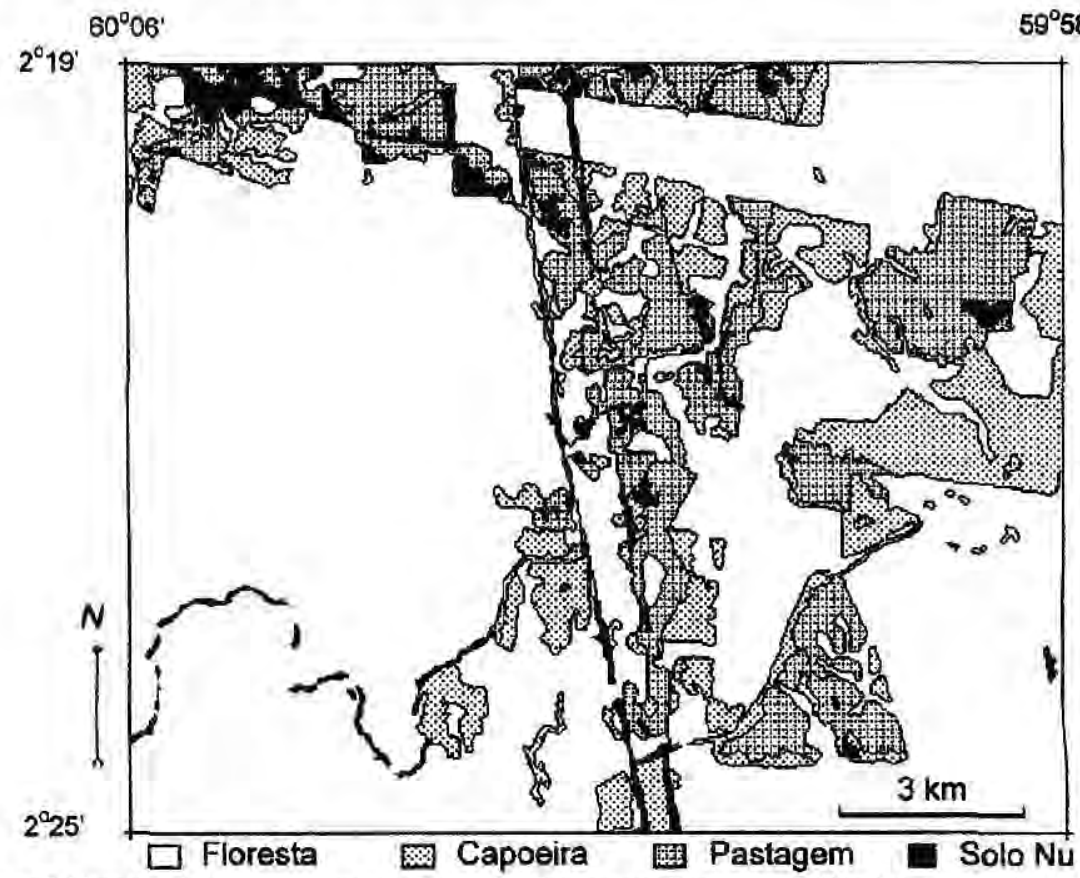

Figura 5. Mapa temático da área de estudo obtido por classificaçâo automática não-supervisionada, a partir de imagens mișturas. 
Tabela 1. Interseções entre as Classes Temáticas do Mapa Fotointerpretado e os Mapas Gerados por Classificação Automática em Imagens Landsat Originais ${ }^{(3)}$ e Convertidas em Imagens Misturas ${ }^{(\mathrm{th})}$.

\begin{tabular}{|c|c|c|c|c|c|}
\hline \multirow{2}{*}{\multicolumn{2}{|c|}{$\begin{array}{c}\text { Mapas Gerados por } \\
\text { Classificação Automática }\end{array}$}} & \multicolumn{4}{|c|}{ Mapa Fotointerpretado de Referência } \\
\hline & & \multirow{2}{*}{$\begin{array}{l}\text { Florestas } \\
133,3 \mathrm{~km}^{2}\end{array}$} & \multirow{2}{*}{$\begin{array}{c}\text { Flor. Secund. } \\
\text { (capoeira) } \\
26,8 \mathrm{~km}^{2}\end{array}$} & \multirow{2}{*}{$\begin{array}{c}\text { Pastagens } \\
25,0 \mathrm{~km}^{2}\end{array}$} & \multirow{2}{*}{$\begin{array}{l}\text { Solos } \\
4,7 \mathrm{~km}^{2}\end{array}$} \\
\hline & & & & & \\
\hline \multirow[t]{2}{*}{ Florestas } & $136,3 \mathrm{~km}^{2(\mathrm{a})}$ & $94,4 \%$ & $3,2 \%$ & $1,6 \%$ & $0,8 \%$ \\
\hline & $136,6 \mathrm{~km}^{2(\mathrm{~b})}$ & $94,7 \%$ & $3,0 \%$ & $1,6 \%$ & $0,6 \%$ \\
\hline \multirow{2}{*}{$\begin{array}{c}\text { Flor. Secund. } \\
\text { (capoeira) }\end{array}$} & $25,8 \mathrm{~km}^{2(a)}$ & $10,2 \%$ & $76,3 \%$ & $12,4 \%$ & $1,1 \%$ \\
\hline & $25,3 \mathrm{~km}^{2(\mathrm{~b})}$ & $10,6 \%$ & $79,0 \%$ & $9,2 \%$ & $0,6 \%$ \\
\hline \multirow[t]{2}{*}{ Pastagens } & $22,9 \mathrm{~km}^{2(\mathrm{a})}$ & $5,4 \%$ & $6,0 \%$ & $77,3 \%$ & $8,1 \%$ \\
\hline & $22,2 \mathrm{~km}^{2}$ (b) & $3,9 \%$ & $9,2 \%$ & $81,1 \%$ & $6,2 \%$ \\
\hline \multirow[t]{2}{*}{ Solos } & $4,7 \mathrm{~km}^{2(a)}$ & $16,2 \%$ & $8,8 \%$ & $38,1 \%$ & $32,3 \%$ \\
\hline & $5,6 \mathrm{~km}^{2(\mathrm{~b})}$ & $7,7 \%$ & $13,4 \%$ & $42,0 \%$ & $41,6 \%$ \\
\hline
\end{tabular}

(capoeiras), 1,6\% de confusão com áreas de pastagens e menos de $1 \%$ com solos nus. Ainda de acordo com esses dados, as áreas ocupadas com Floresta Densa foram superestimadas em pouco mais de $3 \mathrm{~km}^{2}$, em relação ao mapa de referência.

À medida que diminui a área das classes, cai a relação de correspondência espacial entre o mapa fotointerpretado e os mapas gerados por classificação automática. A classe Floresta Secundária que cobre cerca de $13 \%$ da área de estudo, foi subestimada em cerca de $1,0 \mathrm{~km}^{2} \mathrm{e}$ $1,5 \mathrm{~km}^{2}$ nas classificações automáticas das imagens originais e misturas, respectivamente, em relação ao mapa de referência. Os indices de concordância foram $76 \%$ e $79 \%$, enquanto que os índices de confusão foram em torno de $10 \%$, em relação às classes Floresta Densa e Pastagem. Esta confusão entre capoeiras com florestas e pastagens era esperada, uma vez que a capoeira é um estágio intermediário entre a pastagem e a floresta. A classe Pastagem, que também cobre cerca de $13 \%$ da área de estudo, foi subestimada, respectivamente em $2 \mathrm{~km}^{2} \mathrm{e}$ $2,8 \mathrm{~km}^{2}$ com concordância de 77 e $81 \%$ em relação ao mapa de referência. Os indices de confusão variaram entre 4 e $9 \%$ em relação às demais classes. Para a classe Solo $\mathrm{Nu}$, que cobre apenas $3 \%$ da área total, as concordâncias foram de apenas 32 e $42 \%$ em relação ao mapa de referência, mostrando indices de confusão bastante altos com todas as classes, especialmente em relação a classe Pastagem, onde estiveram em torno de $40 \%$. Dada a dificuldade de mapeamento dessa classe via interpretação visual, acredita-se que resultados obtidos pelas classificações automáticas sejam mais corretos, especialmente aqueles obtidos através das imagens misturas, uma vez que elas possuem uma componente especifica para caracterização de solos nus.

O desempenho global considerando a classificação das quatro classes de uso da terra (floresta, capoeira, pastagem e solo nu) foi de $84 \%$ e $90 \%$, 
respectivamente para as classificações das imagens originais e misturas. Quando se considerou apenas a classificação de duas classes i.e. floresta e não-floresta (incluindo como uma única classe capoeira, pastagem e solo nu), o desempenho subiu para $93 \%$ e $94 \%$, respectivamente. Este desempenho refere-se à precisão de mapeamento o que leva em conta não somente a classificação correta de uma determinada categoria mas também sua localização.

Comparando-se os mapas resultantes das classificações automáticas baseadas nas imagens Landsat TM originais e imagens misturas com o mapa de referência obtido por interpretação visual, obteve-se valores da estatística kappa de 0,65 (imagens originais) e 0,77 (imagens misturas) respectivamente, $o$ que indica que houve uma concordância "muito boa" segundo Landis \& Koch (1977) (significativa em torno de 95\% de confiança) entre as classificações automáticas e o mapa de referência.

\section{CONCLUSÕES}

A visão sinóptica e a repetitividade propiciadas pelas imagens orbitais, aliadas às modernas técnicas de processamento digital de imagens, permitem que se façam análises de extensas áreas, obtendo-se resultados com maior rapidez e precisão, se comparados com resultados obtidos por métodos tradicionais de levantamentos, muitas vezes inviáveis em áreas inóspitas como a Amazônia. Talabordagem permite redução substancial de tempo e custos, pela não necessidade de geração e digitalização manual de "overlays".
Técnicas de segmentação e classificação por regiões em imagens $\mathrm{TM} /$ Landsat mostraram-se altamente satisfatórias no mapeamento de unidades de paisagem na Amazônia, com valores de exatidão global entre 84 e $90 \%$, considerando-se quatro classes temáticas de interesse (floresta, capoeira, pastagem e solo nu). Esses valores sobem para 93 e $94 \%$, respectivamente, em imagens Landsat TM originais e misturas se as classes de interesse restringirem-se apenas a áreas de floresta primária e nãofloresta (capoeiras, pastagens, e solos nus), o que muitas vezes, por motivo de simplificação, é o que interessa para modelos climáticos ou de simulação de mudanças de uso da terra.

Com relação à atribuição de limiares no processo de segmentação, entre os diversos pares de limiares testados, o par com valor 12 para o limiar de similaridade e 10 para o de área, foi o que melhor delimitou as classes temáticas de interesse (floresta primária, capoeira, pastagem e solo nu). Valores de limiares mais baixos resultaram em particionamento excessivo, tornando as regiões visualmente confusas, $o$ que dificultou a identificação dos segmentos. Por outro lado, valores mais altos agruparam em um mesmo segmento, áreas distintas, ocasionando a perda total ou parcial de algumas feições.

A técnica de classificação nãosupervisionada por regiões também exige a definição pelo analista de um limiar de aceitação abaixo do qual regiões ou segmentos são agrupados num único segmento. O limiar de $75 \%$ 
foi $o$ que forneceu a melhor classificação da área estudada.

A estatística kappa mostrou que houve concordância significativa $(95 \%$ de confiança) entre as classificações automáticas tanto das imagens originais quanto das imagens misturas e o mapa fotointerpretado de referência. As imagens misturas geraram valor da estatística kappa superior ao das imagensoriginais, indicando que a decomposição das imagens originais em componentes puros tais como vegetação verde, vegetação seca (madeira), solos, e sombra permitiram uma melhor classificação das unidades.

\section{AGRADECIMENTOS}

Aos doutores João Roberto dos Santos e Yosio Edemir Shimabukuro (DSR/INPE) pelas sugestões e comentários a respeito da metodologia utilizada. Ao Dr. John B, Adams (Universidade de Washington, EUA) pela preparação das imagens misturas. Aos doutores Dar Roberts (Universidade da California, Santa Barbara, EUA, Valerie Kapos (Universidade de Cambridge, Reino Unido) e John B. Adams pelo apoio no trabalho de campo.

\section{Bibliografia Citada}

Adams, J. B.; Kapos, V.; Smith, M. O.; Almeida Filho, R.; Gillespie, A. R.; Roberts, D. A. 1990 . A new LANDSAT view of land use in Amazônia. In: International Symposium on Primary Data Acquisition, 1., Commission I, Manaus, AM, 1990. ISPRS. International Archives of Photogrammetric and Remote Sensing. Manaus, ISPRS, v. 28, p.177-185.

Adams, J. B. ; Sabol, D. E.; Kapos, V.; Almeida
Filho, R.; Roberts, D. A.; Smith, M. O.; Gillespie, A. R. 1995. Classification of multispectral images based on fractions of endmembers: application to land-cover change in the Brazilian Amazon. Remote Sensing of Environment, 52(2):137-154.

Alves, D. S.; Moreira, J. C.; Kalil, E. M; Soares, J. V.; Fernandez, O. F.; Almeida, S.; Ortiz, J. D.; Amaral, S. 1996. Mapeamento do uso da terra em Rondônia utilizando técnicas de segmentação e classificação de imagens TM. In: Simpósio Brasileiro de Sensoriamento Remoto, 8., Salvador, 1419 abr. 1996. Anais. São José dos Campos, INPE, SELPER. (Em CD-ROM).

Batista, G. T.; Medeiros, J. S.; Mello, E. M. K.; Moreira, J. C.; Bins, L. S. 1994. A new approach for deforestation assessment. In: Resource and Environmental Monitoring: Rio de Janeiro, ISPRS 1994, v. 30, Part 7a p.170-174. Proceedings of International Symposium organized by VII of the International Society of Photogrammetry and Remote Sensing, Rio de Janeiro, Sept. 26-30, 1994.

Bins, L. S.; Erthal, G. J. ; Fonseca, L. M. G. 1992. Um método de classificação nãosupervisionada por regiões. In: Simpósio Brasileiro de Computação Gráfica e Processamento de Imagens, 5, Águas de Lindóia, 1992. Anais. INPE, São José dos Campos, 1992 , v. 2, p. 65-68.

Cong, P; Howart, P. J. 1990. An assessment of some factors influencing multispectral land-cover classification. Photogrammetric Engineering and Remote Sensing, 56(5):597-603.

Instituto Nacional de Pesquisas Espaciais (INPE) 1996. PRODES: assessment of deforestation in Brazilian Amazônia. INPE, São José dos Campos. junho, 1996. 49 p. (Em CD-ROM).

Landis, J; Koch, G.G. 1977. The measurements of observer agreement for categorial data. Biometrics, 33(3):159-174.

Machado, R. J.; Barbosa, V. C.; Liporace, F. S.; Santos, J. R.; Venturieri, A. 1994. Deforestation monitoring of the Amazon 
region using neural networks - a com parison between different photo-interpreters and networks. In: Resource and Environmental Monitoring: Rio de Janeiro, ISPRS 1994, v. 30, Part 7b p.4955. Proceedings of an International Symposium organized by VII of the International Society of Photogrammetry and Remote Sensing, Rio de Janeiro, Sept. 26-30, 1994.

Medeiros, J. S. 1987. Desenvolvimento metodológico para a deteção de alterações da cohertura vegetal através da análise digital de dados MSS/Landsat. (Dissertação de Mestrado em Sensoriamento Remoto) - Instituto Nacional de Pesquisas Espaciais, São José dos Campos. 81p. (INPE-4123-TDL/262).

Nagao, M.; Matsuyama, T. 1979. Edge preserving smoothing. Computer Graphics and Image Processing, 9(4): 391-407.

Radambrasil, 1978. Radambrasil, Folha SA 20 Manaus, geologia, geomorfologia, pedologia, vegetaçào e uso potencial da terra. Rio de Janeiro, 1978. v.18.
Rosenfield, G.H.; Fitzpatrick-Lins, K. 1986. A coefficient of agreement as a measure of thematic classifications accuracy. Photogrametric Engineering and Remote Sensing, 52(2):223-227.

Santos, J. R.; Venturieri, A; Machado, R. J. 1995. Monitoring land use in Amazonia based on image segmentation and neural networks. In: IGARSS'95 International Geoscience and Remote Sensing Symposium, Firenze, 10-14 July 1995. Quantitative Remote Sensing for Science and Applications. Piscataway, IEEE, v.3, p.108-111.

Star, J.; Estes, J. 1990. Geographic Information Sysiem: an introduction. Prentice Hall, Englewood Cliffs, New Jersey.

Tardin, A. T.; Cunha, R. P. 1989. Avaliação da alteração da cobertura florestal na Amazónia Legal utilizando sensoriamento remoto orbital. São José dos Campos, INPE, 43p. (INPE-5010-RPE/607). 